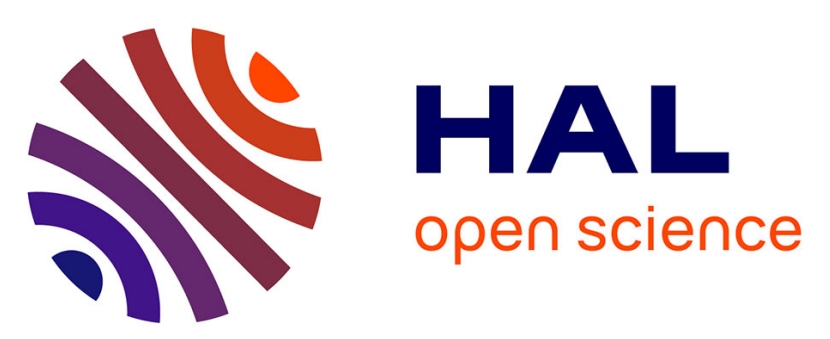

\title{
Obtaining a Triangular Matrix by Independent Row-Column Permutations
}

\author{
Guillaume Fertin, Irena Rusu, Stéphane Vialette
}

\section{To cite this version:}

Guillaume Fertin, Irena Rusu, Stéphane Vialette. Obtaining a Triangular Matrix by Independent Row-Column Permutations. 26th International Symposium on Algorithms and Computation, Dec 2015, Nagoya, France. hal-01189621

\section{HAL Id: hal-01189621 \\ https://hal.science/hal-01189621}

Submitted on 1 Sep 2015

HAL is a multi-disciplinary open access archive for the deposit and dissemination of scientific research documents, whether they are published or not. The documents may come from teaching and research institutions in France or abroad, or from public or private research centers.
L'archive ouverte pluridisciplinaire HAL, est destinée au dépôt et à la diffusion de documents scientifiques de niveau recherche, publiés ou non, émanant des établissements d'enseignement et de recherche français ou étrangers, des laboratoires publics ou privés. 


\title{
Obtaining a Triangular Matrix by Independent Row-Column Permutations
}

\author{
Guillaume Fertin ${ }^{1}$, Irena Rusu ${ }^{1}$, and Stéphane Vialette ${ }^{2}$ \\ 1 LINA UMR CNRS 6241, Université de Nantes, France \\ \{guillaume.fertin, irena.rusu\}@univ-nantes.fr \\ 2 Université Paris-Est, LIGM (UMR 8049), CNRS, UPEM, ESIEE Paris, ENPC, \\ F-77454, Marne-la-Vallée, France \\ vialette@univ-mlv.fr
}

\begin{abstract}
Given a square $(0,1)$-matrix $A$, we consider the problem of deciding whether there exists a permutation of the rows and a permutation of the columns of $A$ such that after carrying out these permutations, the resulting matrix is triangular. The complexity of the problem was posed as an open question by Wilf [7] in 1997. In 1998, DasGupta et al. [3] seemingly answered the question, proving it is NP-complete. However, we show here that their result is flawed, which leaves the question still open. Therefore, we give a definite answer to this question by proving that the problem is NP-complete. We finally present an exponential-time algorithm for solving the problem.
\end{abstract}

\section{Introduction}

In his contribution in the tribute to the late Professor Erdös [7], Wilf posed the following question: "Let $A$ be $a m \times n$ matrix of 0 's and 1 's. Consider the computational problem: do there exist permutations $P$ of the rows of $A$, and $Q$, of the columns of $A$ such that after carrying out these permutations, $A$ is triangular? The question we ask concerns the complexity of the problem. Is this problem NP-complete? Or, does there exist a polynomial-time algorithm for doing it?" As noted by Wilf, this problem is strongly related to job scheduling with precedence constraints, a well-known problem in theoretical computer science. The present paper is devoted to giving an answer to this question.

A square matrix is called lower triangular if all the entries above the main diagonal are zero. Similarly, a square matrix is called upper triangular if all the entries below the main diagonal are zero. A triangular matrix is one that is either lower triangular or upper triangular. Because matrix equations with triangular matrices are easier to solve, they are very important in linear algebra and numerical analysis. We refer the reader to [4] for a further discussion.

For an arbitrary square matrix $A$, it is well-known in linear algebra that there exists an invertible matrix $S$ such that $S^{-1} A S$ is upper triangular. We focus here, however, on permutation matrices. Recall that a permutation matrix is a square matrix obtained from the same size identity matrix by a permutation of rows. A product of permutation matrices is again a permutation matrix and 
the inverse of a permutation matrix is again a permutation matrix. In fact, for any permutation matrix $P, P^{-1}=P^{T}$.

This paper is organized as follows. In Section 2, we provide the basic material needed for this paper. Section 3 is devoted to proving hardness of determining whether a square $(0,1)$-matrix is permutation equivalent triangular, i.e. whether it can be transformed into a triangular matrix by independent row and column permutations. In Section 4, we give some properties of permutation equivalent triangular matrices (or pet matrices, for short) and present an exponential-time algorithm to determine whether a matrix is a pet matrix. The paper concludes with suggestions for further research directions.

\section{Notations}

For any positive integer $n$, denote $[n]=\{1,2, \ldots, n\}$. Let $A=\left[a_{i, j}\right], 1 \leq i \leq m$ and $1 \leq j \leq n$, be a matrix of $m$ rows and $n$ columns. In the case that $m=n$ then the matrix is square of order $n$. It is always assumed that the entries of the matrix are elements of some underlying field $F$. It is convenient to refer to either a row or a column of the matrix as a line of the matrix. We use the notation $A^{T}$ for the transpose of matrix $A$. We always designate a zero matrix by $\mathbf{0}$, a matrix with every entry equal to 1 by $J$, and the identity matrix of order $n$ by $I$. In order to emphasize the size of these matrices we sometimes include subscripts. Thus $J_{m, n}$ denotes the all 1's matrix of size $m$ by $n$, and this is abbreviated to $J_{n}$ if $m=n$. Notations $\mathbf{0}_{m, n}, \mathbf{0}_{n}$ and $I_{n}$ are similarly defined. In displaying a matrix we often use $*$ to designate a submatrix of no particular structure. Two matrices $A$ and $B$ are said to be permutation equivalent, denoted by $A \sim B$, if there exist permutation matrices $P$ and $Q$ of suitable sizes such that $B=P A Q$.

We will be greatly concerned with matrices whose entries consist exclusively of the integers 0 and 1 . Such matrices are referred to as $(0,1)$-matrices. For a $(0,1)$-matrix $A$, we let $\omega(A)$ stand for the number of 1 's in $A$. A square matrix $A=\left[a_{i, j}\right]$ of order $n$ is said to be lower left triangular if it has only 0's above the main diagonal (i.e. $a_{i, j}=0$ for $1 \leq i<j \leq n$. We write $\triangle_{n}$ for the lower left triangular $(0,1)$-matrix whose 0 's are exclusively above the main diagonal. For two matrices $A=\left[a_{i, j}\right]$ and $B=\left[b_{i, j}\right]$ of size $m$ by $n$, we write $A \leq B$ if $a_{i, j} \leq b_{i, j}$ for $1 \leq i \leq m$ and $1 \leq j \leq n$, so that a square matrix $A$ of order $n$ is lower left triangular if $A \leq \triangle_{n}$. In the context of permutation equivalent matrices, we will sometimes not be interested in any particular orientation of a triangular matrix and forget about any specific orientation such as "lower left". Furthermore, for readability, a matrix which is permutation equivalent to a triangular matrix is said to be a pet matrix. The row sum vector $\mathcal{R}(A)=\left[r_{1} r_{2} \ldots r_{m}\right]$ and the column sum vector $\mathcal{C}(A)=\left[c_{1} c_{2} \ldots c_{n}\right]$ of $A$ are defined by $r_{i}=\sum_{1 \leq j \leq n} a_{i, j}$ for $1 \leq i \leq m$ and $c_{j}=\sum_{1<i<m} a_{i, j}$ for $1 \leq j \leq n$. The row sum vector $\mathcal{R}(A)$ (resp. column sum vector $\mathcal{C}(A)$ ) is stepwise bounded if $\left|\left\{i: r_{i} \leq k\right\}\right| \geq k$ (resp. $\left.\left|\left\{j: c_{j} \leq k\right\}\right| \geq k\right)$ for $1 \leq k \leq n$. It is clear that if a $(0,1)$-matrix $A$ is a pet matrix then both $\mathcal{R}(A)$ and $\mathcal{C}(A)$ are stepwise bounded. 
The permanent of $A=\left[a_{i, j}\right]$ is defined as $\operatorname{per}(A)=\sum_{\left(j_{1}, j_{2}, \ldots, j_{n}\right) \in S_{n}} a_{1, j_{1}} a_{2, j_{2}} \ldots a_{n, j_{n}}$ where the summation is over all permutations $\left(j_{1}, j_{2}, \ldots, j_{n}\right)$ of $[n]$. Observe that, unlike the determinant, we do not put a minus sign in front of some of the terms in the summation. Of particular importance, the permanent does not change if we permute the rows of $A$ and permute the columns of $A$.

Let $A=\left[a_{i, j}\right]$ be an $m$ by $n$ matrix. For convenience, for a set $K \subseteq[m]$ we will write $\bar{K}$ for the set $[m] \backslash K$. Let $K=\left\{i_{1}, i_{2}, \ldots, i_{k}\right\}$ be a set of $k$ elements with $K \subseteq[m]$, and let $L=\left\{j_{1}, j_{2}, \ldots, j_{l}\right\}$ be a set of $l$ elements with $L \subseteq[n]$. The sets $K$ and $L$ designate a collection of row indices and column indices, respectively, of the matrix $A$, and the $k$ by $l$ submatrix determined by them is denoted $A[K, L]$.

Let $X=\left\{x_{i}: 1 \leq i \leq n\right\}$ be a non-empty set of $n$ elements, that we call an $n$-set. Let $\mathcal{S}=\left(S_{i}: 1 \leq i \leq m\right)$ be $m$ not necessarily distinct subsets of the $n$-set $X$. We refer to this collection of subsets of an $n$-set as a configuration of subsets. We set $a_{i, j}=1$ if $x_{j} \in S_{i}$, and $a_{i, j}=0$ if $x_{i} \notin S_{i}$. The resulting (0,1)-matrix $A=\left[a_{i, j}\right], 1 \leq i \leq m$ and $1 \leq j \leq n$ of size $m$ by $n$ is the incidence matrix for the configuration of subsets $\mathcal{S}$ of the $n$-set $X$. The 1 s in row $\alpha_{i}$ of $A$ display the elements in the subset $S_{i}$, and the 1's in column $\beta_{j}$ display the occurrences of $x_{j}$ among the subsets. Let $\mathcal{S}=\left(S_{i}: 1 \leq i \leq n\right)$ be a configuration of subsets of some ground $n$-set $X$. A bijective mapping $\varphi: \mathcal{S} \rightarrow[n]$ is said to be a stepwise bounded labeling (or sbl for short) of $\mathcal{S}$ if $\left|\bigcup_{\varphi\left(S_{j}\right) \leq i} S_{j}\right| \leq i$ for $1 \leq i \leq n$.

\section{Answering Wilf's question}

We prove in this section that, given a square $(0,1)$-matrix $A$, deciding whether there exists a permutation matrix $P$ and a permutation matrix $Q$ of suitable size such that $P A Q$ is triangular is NP-complete.

\subsection{Disproving a previous related result}

Before giving our proof, it is worth mentioning that the following problem (called $\operatorname{LBQIS}(n, k)$ and rephrased to fit the context of this paper) is claimed to be NP-complete in [3]: Given a (0,1)-matrix of order $n$ and positive integer $k \leq n$, do there exist permutation matrices $P$ and $Q$ such that $P A Q=\left[\begin{array}{ll}A_{1,1} & A_{1,2} \\ A_{2,1} & A_{2,2}\end{array}\right]$ with $A_{1,2}$ a square lower triangular matrix of size $k$ by $k$ ? It is not very difficult to find a polynomial transformation from LBQIS to Wilf's question, which would prove the NP-completeness of the latter. Just add $n-k$ empty rows and $n-k$ empty columns to matrix $A$ to obtain a new matrix $A^{\prime}$. Now, notice that each submatrix $A_{1,2}$ in a solution for LBQIS may be completed with the $n-k$ empty rows put before row 1 of $A_{1,2}$ and with the $n-k$ empty columns put after column $k$ of $A_{1,2}$ to yield a solution for the instance $A^{\prime}$ in Wilf's question, and viceversa.

Unfortunately, paper [3] contains a serious flaw in the proof. To fix things, note that in [3] LBQIS is stated in terms of bipartite graphs, for which matrix $A$ is the reduced adjacency matrix. Then, $\operatorname{LBQIS}(n, k)$ is proved $\mathbf{N P}$-complete 
by reduction from another problem on bipartite graphs called $\operatorname{LBIS}(n, k)$, using the so-called Rearrangement Lemma (Lemma 3.5 in [3]). Two affirmations in the proof of this lemma are contradicted by the following example. Let $G$ be the graph (input for LBIS) with vertices $U=\{i \mid 1 \leq i \leq 4\}$ and $V=\{i \mid 1 \leq$ $i \leq 4\}$, whose edges are $(1,1),(2,1),(2,2),(3,2),(3,4),(4,3)$ and $(4,4)$. Thus, $n=4$. Define $k=1$. Let $G^{\prime}$ be the input graph for LBQIS built as in [3], and $k^{\prime}=k^{2}+k=2$. Then the vertex subset $U^{\prime} \cup V^{\prime}$ of $G^{\prime}$, with $U^{\prime}=\{[2,4],[1,2]\}$ and $V^{\prime}=\{[1,3],[2,2]\}$ is a solution of LBQIS of size $k^{\prime}$ for which the assumption on the first line of the Rearrangement Lemma's proof is false. Also, the vertex subset $U^{\prime} \cup V^{\prime}$ of $G^{\prime}$, with $U^{\prime}=\{[1,1],[2,1]\}$ and $V^{\prime}=\{[1,2],[1,3]\}$ is a solution of LBQIS of size $k^{\prime}$ for which the second affirmation in the same lemma ("clearly $\left.q_{1} \leq p_{1}{ }^{\prime \prime}\right)$ is also false.

\subsection{Our NP-completeness proof for Wilf's question}

We present our results in terms of sbl for configurations of subsets. The rationale for considering sbl for configurations of subsets stems from the following lemma.

Lemma 1. Let $\mathcal{S}=\left(S_{i}: 1 \leq i \leq n\right)$ be a configuration of subsets of some ground $n$-set, and let $A$ be the corresponding incidence matrix. There exist permutation matrices $P$ and $Q$ of order $n$ such that $P A Q \leq \triangle_{n}$ if and only if there exists an sbl of $\mathcal{S}$.

We need to focus our attention on a special type of sbl. Call a bijective mapping $\varphi: \mathcal{S} \rightarrow[n]$ normalized if $\varphi$ maps the identical subsets of elements of $\mathcal{S}$ to a set of consecutive integers. Most of the interest in normalized bijective labelings stems from the following intuitive lemma.

Lemma 2. Let $\mathcal{S}=\left(S_{i}: 1 \leq i \leq n\right)$ be a configuration of subsets of some ground $n$-set. If there exists an sbl of $\mathcal{S}$ then there exists a normalized sbl of $\mathcal{S}$.

We are now ready to prove that deciding whether there exists an sbl of some configuration of subsets is NP-complete thereby proving that deciding whether a square $(0,1)$-matrix is a pet matrix is $\mathbf{N P}$-complete as well. The proof proceeds by a reduction from the 3SAT problem - which is a known NP-complete problem [2]. Let an arbitrary instance of the $3 \mathrm{SAT}$ problem be given by a $3 \mathrm{CNF}$ formula $\phi=c_{1} \vee c_{2} \vee \ldots \vee c_{m}$ over variables $x_{1}, x_{2}, \ldots, x_{n}$. Our construction is divided into two steps: (1) construction of a (polynomial size) ground set $\mathbf{X}$ and (2) construction of a configuration of subsets $C$ of the ground set $\mathbf{X}$. Throughout the proof, parts of the ground set $\mathbf{X}$ are written as capital bold letters $(\mathbf{V}, \mathbf{T}, \mathbf{F}, \ldots)$ and subsets of the configuration are written with capital calligraphic letters $\left(\mathcal{V}_{i}, \mathcal{T}_{i}, \mathcal{F}_{i}, \ldots\right)$ 


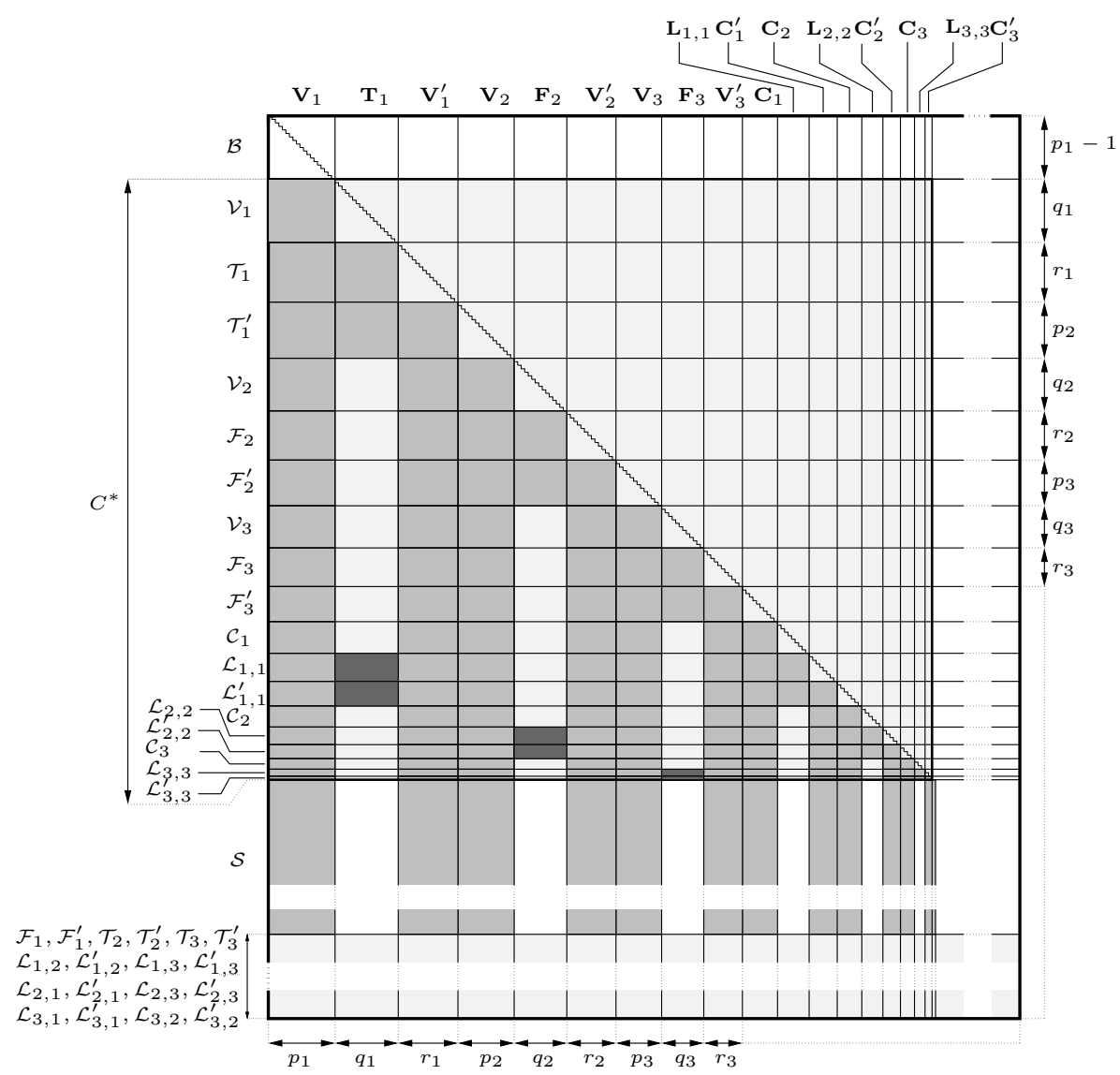

Fig. 1. Illustration of the construction for the 3CNF formula $\phi=\left(x_{1} \vee x_{2} \vee x_{3}\right) \wedge\left(\bar{x}_{1} \vee\right.$ $\left.\bar{x}_{2} \vee x_{3}\right) \wedge\left(\bar{x}_{1} \vee x_{2} \vee \bar{x}_{3}\right)$. Identical subsets are not distinguishable in our representation. A satisfying truth assignment is given by $f\left(x_{1}\right)=$ TRUE, $f\left(x_{2}\right)=$ FALSE and $f\left(x_{3}\right)=$ FALSE. For sake of clarity, neither the ground set $\mathbf{X}$ nor the collection of subsets $C$ is fully represented.

To begin with, define $p_{i}=3(n+m+1-i)+2, q_{i}=3(n+m+1-i)+1$ and $r_{i}=3(n+m+1-i)$ for $1 \leq i \leq n+m$. Furthermore, define $p_{n+m+1}=1, K=$ $\sum_{i=1}^{n} q_{i}+2 \sum_{i=n+1}^{n+m} q_{i}$ and $L=\sum_{i=1}^{n+m}\left(p_{i+1}+r_{i}\right)$. Let us now define the ground set $\mathbf{X}$. Consider the pairwise disjoint sets defined as follows: $\mathbf{V}_{i}=\left\{v_{i, j} \mid 1 \leq j \leq p_{i}\right\}$, $\mathbf{V}_{i}^{\prime}=\left\{v_{i, j}^{\prime} \mid 1 \leq j \leq r_{i}\right\}, \mathbf{T}_{i}=\left\{t_{i, j} \mid 1 \leq j \leq q_{i}\right\}, \mathbf{F}_{i}=\left\{f_{i, j} \mid 1 \leq j \leq q_{i}\right\}$ for $1 \leq i \leq n$. Furthermore, define $\mathbf{C}_{i}=\left\{c_{i, j} \mid 1 \leq j \leq p_{n+i}\right\} \mathbf{C}_{i}^{\prime}=\left\{c_{i, j}^{\prime} \mid 1 \leq\right.$ $\left.j \leq r_{n+i}\right\}$ for $1 \leq i \leq m$, and $\mathbf{L}_{i, k}=\left\{\ell_{i, k, j} \mid 1 \leq j \leq q_{n+i}\right\}$ for $1 \leq i \leq m$ and $1 \leq k \leq 3$. Finally, define $\mathbf{S}=\{s\}$. For simplicity of notation, write $\overline{\mathbf{V}}=$ $\bigcup_{1 \leq i \leq n} \mathbf{V}_{i}, \mathbf{V}^{\prime}=\bigcup_{1 \leq i \leq n} \mathbf{V}_{i}^{\prime}, \mathbf{T}=\bigcup_{1 \leq i \leq n} \mathbf{T}_{i}, \mathbf{F}=\bigcup_{1 \leq i \leq n} \mathbf{F}_{i}, \mathbf{C}=\bigcup_{1 \leq i \leq m} \mathbf{C}_{i}$, $\mathbf{C}^{\prime}=\bigcup_{1 \leq i \leq m} \mathbf{C}_{i}^{\prime}$, and $\mathbf{L}_{i}=\bigcup_{1 \leq k \leq 3} \overline{\mathbf{L}}_{i, k}$ for $1 \leq i \leq m$ and $\mathbf{L}=\bigcup_{1<i<m} \mathbf{L}_{i}$. Informally, elements of $\mathbf{V} \cup \mathbf{V}^{\prime}$ are associated to variables, elements of $\mathbf{T} \cup \mathbf{F}$ are 
associated to literals, elements of $\mathbf{C} \cup \mathbf{C}^{\prime}$ are associated to clauses, elements of $\mathbf{L}$ are associated to literals in clauses and $\mathbf{S}$ is a separator set. The ground set $\mathbf{X}$ of our construction is defined to be $\mathbf{X}=\mathbf{V} \cup \mathbf{V}^{\prime} \cup \mathbf{T} \cup \mathbf{F} \cup \mathbf{C} \cup \mathbf{C}^{\prime} \cup \mathbf{L} \cup \mathbf{S}$.

Having defined the ground set $\mathbf{X}$, we now turn to the detailed construction of a configuration of subsets $C$ of $\mathbf{X}$. For sake of clarity, this will be divided into several steps. First, each variable $x_{i}, 1 \leq i \leq n$, is associated to identical subsets $\mathcal{V}_{i, j}, 1 \leq j \leq q_{i}$, in $C$. These subsets are defined as follows: $\mathcal{V}_{i, j}=$ $\left(\bigcup_{1 \leq k \leq i} \mathbf{V}_{k}\right) \cup\left(\bigcup_{1 \leq k \leq i-1} \mathbf{V}_{k}^{\prime}\right)$ for $1 \leq i \leq n$ and $1 \leq j \leq q_{i}$. Let us denote by $\mathcal{V}_{i}, 1 \leq i \leq n$, the collection $\left(\mathcal{V}_{i, j} \mid 1 \leq j \leq q_{i}\right)$. Next, each (positive) literal $x_{i}, 1 \leq i \leq n$, is associated to identical subsets $\mathcal{T}_{i, j}, 1 \leq j \leq r_{i}$, and to identical subset $\mathcal{T}_{i, j}^{\prime}, 1 \leq j \leq p_{i+1}$. These subsets are defined as follows: $\mathcal{T}_{i, j}=\mathbf{T}_{i} \cup\left(\bigcup_{1 \leq k \leq i} \mathbf{V}_{k}\right) \cup\left(\bigcup_{1 \leq k \leq i-1} \mathbf{V}_{k}^{\prime}\right)$ for $1 \leq i \leq n$ and $1 \leq j \leq r_{i}$, and $\mathcal{T}_{i, j}^{\prime}=\mathbf{T}_{i} \cup\left(\bigcup_{1 \leq k \leq i} \mathbf{V}_{k}\right) \cup\left(\bigcup_{1 \leq k \leq i} \mathbf{V}_{k}^{\prime}\right)$ for $1 \leq i \leq n$ and $1 \leq j \leq p_{i+1}$. Of course, a similar construction of subsets applies for the negation $\bar{x}_{i}$ of each variable $x_{i}$, i.e., $\mathcal{F}_{i, j}=\mathbf{F}_{i} \cup\left(\bigcup_{1 \leq k \leq i} \mathbf{V}_{k}\right) \cup\left(\bigcup_{1 \leq k \leq i-1} \mathbf{V}_{k}^{\prime}\right)$ for $1 \leq i \leq n$ and $1 \leq j \leq r_{i}$, and $\mathcal{F}_{i, j}^{\prime}=\mathbf{F}_{i} \cup\left(\bigcup_{1 \leq k \leq i} \mathbf{V}_{k}\right) \cup\left(\bigcup_{1 \leq k \leq i} \mathbf{V}_{k}^{\prime}\right)$ for $1 \leq i \leq n$ and $1 \leq j \leq p_{i+1}$. For readability, write $\mathcal{T}_{i}=\left(\mathcal{T}_{i, j} \mid 1 \leq j \leq r_{i}\right), \mathcal{T}_{i}^{\prime}=\left(\mathcal{T}_{i, j}^{\prime} \mid\right.$ $\left.1 \leq j \leq p_{i+1}\right), \mathcal{F}_{i}=\left(\mathcal{F}_{i, j} \mid 1 \leq j \leq r_{i}\right)$ and $\mathcal{F}_{i}^{\prime}=\left(\mathcal{F}_{i, j}^{\prime} \mid 1 \leq j \leq p_{i+1}\right)$ for $1 \leq i \leq n$. Note that the following (strict) inclusions hold for all $1 \leq i \leq n$, $1 \leq j_{1} \leq q_{i}, 1 \leq j_{2} \leq r_{i}$ and $1 \leq j_{3} \leq p_{i+1}$ : (i) $\mathcal{V}_{i, j_{1}} \subset \mathcal{T}_{i, j_{2}} \subset \mathcal{T}_{i, j_{3}}^{\prime}$ and (ii) $\mathcal{V}_{i, j_{1}} \subset \mathcal{F}_{i, j_{2}} \subset \mathcal{F}_{i, j_{3}}^{\prime}$. We now turn to the $m$ clauses of the 3CNF formula. Each clause $c_{i}, 1 \leq i \leq m$, is associated to identical subsets $\mathcal{C}_{i, j}, 1 \leq j \leq q_{n+i}$. These subsets are defined as follows: $\mathcal{C}_{i, j}=\mathbf{V} \cup \mathbf{V}^{\prime} \cup\left(\cup_{1 \leq k \leq i} \mathbf{C}_{k}\right) \cup\left(\cup_{1 \leq k \leq i-1} \mathbf{C}_{k}^{\prime}\right)$ for $1 \leq i \leq m$ and $1 \leq j \leq q_{n+i}$. Let us denote by $\mathcal{C}_{i}, 1 \leq i \leq m$, the collection $\left(\mathcal{C}_{i, j} \mid 1 \leq j \leq q_{n+i}\right)$. It is easily seen that $\mathcal{V}_{i, j_{1}} \subset \mathcal{C}_{k, j_{2}}$ for all $1 \leq i \leq n$, $1 \leq j_{1} \leq q_{i}, 1 \leq k \leq m$ and $1 \leq j_{2} \leq q_{n+k}$.

Now, we consider the only part of the construction that depends on which literal occurs in which clauses. Denote by $\lambda_{i, k}$ the $k$-th literal of clause $c_{i}$, that is write $c_{i}=\lambda_{i, 1} \vee \lambda_{i, 2} \vee \lambda_{i, 3}$ for $1 \leq i \leq m$, where each $\lambda_{i, k}$ is a variable or its negation. The $k$-th literal, $1 \leq k \leq 3$, of each clause $c_{i}, 1 \leq i \leq m$, is associated to identical subsets $\mathcal{L}_{i, k, j}, 1 \leq j \leq r_{n+i}$, and to identical subsets $\mathcal{L}_{i, k, j}^{\prime}, 1 \leq j \leq p_{n+i+1}$. These subsets are defined as follows: $\mathcal{L}_{i, k, j}=\mathbf{V} \cup \mathbf{V}^{\prime} \cup$ $\mathbf{A}_{k} \cup \mathbf{L}_{i, k} \cup\left(\bigcup_{1 \leq \ell \leq i} \mathbf{C}_{\ell}\right) \cup\left(\cup_{1 \leq \ell \leq i-1} \mathbf{C}_{\ell}^{\prime}\right)$ for $1 \leq i \leq m, 1 \leq j \leq r_{n+i}$ and $1 \leq k \leq 3$ and $\mathcal{L}_{i, k, j}^{\prime}=\mathbf{V} \cup \mathbf{V}^{\prime} \cup \mathbf{A}_{k} \cup \mathbf{L}_{i, k} \cup\left(\cup_{1 \leq \ell \leq i} \mathbf{C}_{\ell}\right) \cup\left(\cup_{1 \leq \ell \leq i} \mathbf{C}_{\ell}^{\prime}\right)$ for $1 \leq i \leq m, 1 \leq j \leq p_{n+i+1}$ and $1 \leq k \leq 3$, where $\mathbf{A}_{k}=\mathbf{T}_{\ell}$ if $\lambda_{i, k}=x_{\ell}$ and $\mathbf{A}_{k}=\mathbf{F}_{\ell}$ if $\lambda_{i, k}=\bar{x}_{\ell}$. For the sake of clarity, write $\mathcal{L}_{i, k}=\left(\mathcal{L}_{i, k, j} \mid 1 \leq j \leq r_{n+i}\right)$ and $\mathcal{L}_{i, k}^{\prime}=\left(\mathcal{L}_{i, k, j}^{\prime} \mid 1 \leq j \leq p_{n+i+1}\right)$ for $1 \leq i \leq m$ and $1 \leq k \leq 3$. Again, observe that $\mathcal{C}_{i, j_{1}} \subset \mathcal{L}_{i, k, j_{2}} \subset \mathcal{L}_{i, k, j_{2}}$ for all $1 \leq i \leq m, 1 \leq j_{1} \leq q_{n+i}, 1 \leq j_{2} \leq r_{n+i}$, $1 \leq j_{3} \leq p_{n+i+1}$ and $1 \leq k \leq 3$.

Our construction ends with $p_{1}+K-1$ utility subsets. These subsets will be partitioned into two separate classes according to their indented function: 
bootstrap subsets and separator subsets. First, $C$ contains identical bootstrap subsets $\mathcal{B}_{i}, 1 \leq i \leq p_{1}-1$, defined as follows: $\mathcal{B}_{i}=\emptyset$ for $1 \leq i \leq p_{1}-1$. The idea is to force any sbl to map the $p_{1}-1$ empty sets of $\mathcal{B}$ to the first $p_{1}-1=3(n+m)+1$ integers. Indeed, it is easily seen that all the above defined subsets of the configuration of subsets $C$ but those of $\mathcal{B}$ contain at least $p_{1}$ elements and hence cannot be mapped to an integer $i \leq p_{1}-1$ in any sbl of $C$. Second, $C$ contains identical separator subsets $\mathcal{S}_{i}, 1 \leq i \leq K$, defined by: $\mathcal{S}_{i}=\mathbf{V} \cup \mathbf{V}^{\prime} \cup \mathbf{C} \cup \mathbf{C}^{\prime} \cup \mathbf{S}$ for $1 \leq i \leq K$. The rationale of these subsets is that we need a separator between subsets in $C$ corresponding to a satisfying truth assignment $f$ for the $3 \mathrm{CNF}$ formula $\phi$ and garbage subsets of $C$, that is subsets not involved in the satisfying truth assignment $f$. For simplicity, let us denote by $\mathcal{B}$ the collection $\left(\mathcal{B}_{i} \mid 1 \leq i \leq p_{1}-1\right)$ and by $\mathcal{S}$ the collection $\left(\mathcal{S}_{i} \mid 1 \leq i \leq K\right)$. Clearly our construction can be carried on in polynomial time: indeed, we have $|\mathbf{X}|=O\left(m^{2}+n^{2}\right)$ and $|C|=O\left(m^{2}+n^{2}\right)$.

Lemma 3. There exists a satisfying truth assignment $f$ for $\phi$ if and only if there exists an sbl of the configuration of subsets $C$ of the ground set $\mathbf{X}$.

The key elements of the proof are as follows. First, it is crucial to focus on solutions that map identical subsets of elements of $\mathcal{S}$ to a set of consecutive elements (see Lemma 2). Second, the general shape of the solution is largely guided by the construction. Indeed, the empty subsets have to be placed first, followed by subsets corresponding to literals (either the positive or the negative literal of each variable has been chosen) and next by subsets corresponding to clauses (one satisfying literal of each clause is chose). Finally the separator subsets have to be placed, with the result that (thanks to the large polynomial number of such subsets) the remaining subsets can be placed in any order without violating the sought sbl property. The reader is invited to consider Figure 1 for a schematic illustration of the reduction. We now briefly discuss, in an informal way, the two key arguments that are used in the proof. First, the whole procedure is, to some extent, similar to the accounting method used in amortized complexity analysis. Indeed, one might view the operation of placing a set (one after the other) as the process of charging some customer, the cost being the number of new elements that are introduced. With this metaphor in mind, notice that we do not charge when a subset does not introduce any new element, so that the leftover amount can be stored as "credit". When we place a new subset that does introduce some new elements, we can use the "credit" stored to pay for the cost of the operation. Second, when a subset uses the "credit" stored to pay the cost of introducing new elements, the following invariants can be shown to hold true: (i) it uses all the available credit and (ii) it does not allow to accumulate (it should be now clear that consecutive identical subsets do allow for accumulating credit) as much credit as it has consumed, thereby proving that subsets introduce less and less new elements as we progress adding subsets one after the other.

The main result of this paper can now be stated.

Theorem 1. Let $A$ be a $(0,1)$-matrix. Deciding whether $A$ is a pet matrix is NP-complete. 


\section{Exponential-time algorithm}

We present here an exponential-time algorithm for deciding whether a given a $(0,1)$-matrix $A$ of order $n$ is a pet matrix. We start by presenting some basic properties of square $(0,1)$-matrices that can be transformed into some triangular matrix by row and column independent permutations to help solving involved algorithmic issues. We of course focus of polynomial-time checkable properties.

We first focus on the permanent of a square $(0,1)$-matrix. A well-known result (see e.g. [1]) states that for a $(0,1)$-matrix $A$ of order $n$, one has $\operatorname{per}(A)=1$ if and only if the lines of $A$ may be permuted to yield a triangular matrix with 1 's in the $n$ main diagonal positions and 0's above the mail diagonal. This theorem amounts to saying that $\operatorname{per}(A)=1$ if and only if there exist permutation matrices $P$ and $Q$ such that $I \leq P A Q \leq \triangle$. As shown in the following lemma, $\operatorname{per}(A)=1$ is certainly a threshold value in our context.

Lemma 4. Let $A$ be $(0,1)$-matrix. If $A$ is a pet matrix then $\operatorname{per}(A) \leq 1$.

Notice that deciding $\operatorname{per}(A) \leq 1$ for $(0,1)$-matrices of order $n$ reduces to computing at most $n+1$ perfect matchings in bipartite graphs [1], and hence the above test is $O\left(n^{3} \sqrt{n}\right)$ time as the Hopcroft-Karp algorithm for computing a maximum matching in a bipartite graph $B=(V, E)$ runs in $O(|E| \sqrt{|V|})[6]$.

Next, it is a simple matter to check that if a $(0,1)$-matrix $A$ of order $n$ is a pet matrix, then it contains at most $\frac{1}{2} n(n+1) 1$ 's $\left(\right.$ i.e., $\left.\omega(A) \leq \frac{1}{2} n(n+1)\right)$. The following lemma gives a lower bound.

Lemma 5. Let $A$ be $(0,1)$-matrix of order $n, n \geq 2$. If $A$ contains at most $n+1$ 1 's, then $A$ is a pet matrix.

Notice that, albeit not very impressive, Lemma 5 is tight as the square matrix $\left[\begin{array}{cc}I_{n-2} & \mathbf{0}_{n-2,2} \\ \mathbf{0}_{2, n-2} & J_{2}\end{array}\right]$ of order $n$ has $n-2+4=n+21$ 's and is not a pet matrix.

Finally, the following trivial lemma gives another condition that helps improving the running time of the algorithm in practice.

Lemma 6. Let $A$ be $(0,1)$-matrix of order $n$ and $D$ the directed graph associated to $A$ (i.e., the adjacency matrix of $D$ is $A$ ). If the digraph $D$ is acyclic (regardless self-loops), then $A$ is a pet matrix.

We now turn to presenting the exponential-time algorithm. The simplest exhaustive algorithm considers every possible pairs of permutation matrices $(P, Q)$ yielding a $O\left((n !)^{2} \cdot \operatorname{poly}(n)\right)$ time algorithm. However, according to Lemma 1 , it is enough to consider every permutation matrix $P$ of order $n$ and check whether the first $i, 1 \leq i \leq n$, rows of $P A$ have 1 's in at most $i$ columns. This observation yields a $O(n ! \cdot \operatorname{poly}(n))$ time algorithm. We propose here another exhaustive algorithm that improves on the $O(n ! \cdot \operatorname{poly}(n))$ time algorithm. The basic idea is to recursively split into smaller submatrices, instead of enumerating all permutations. For a $(0,1)$-matrix $A$ of order $n$, we consider every possible set $R$ of $\lceil n / 2\rceil$ rows of $A$ and every possible set of $\lceil n / 2\rceil$ columns $C$ of $A$, and check 


$$
P A Q=\left[\begin{array}{cc}
A_{1} & \mathbf{0} \\
* & A_{2}
\end{array}\right]
$$

(a) Even
$P A Q=\left[\begin{array}{ccc}A_{1} & \mathbf{0} & 0 \\ * & 1 & 0 \\ * & * & A_{2}\end{array}\right]$

(b) Odd and one 1
$P A Q=\left[\begin{array}{ccc}A_{1} & \mathbf{0} & \mathbf{0} \\ * & 0 & \mathbf{0} \\ * & * & A_{2}\end{array}\right]$

(c) Odd and zero 1

Fig. 2 .

whether these lines induce a zero matrix (or a matrix with at most one 1 in case the matrix has odd order; details follow).

If $n$ is even, we let $P$ and $Q$ be two permutation matrices that put the rows in $R$ at the first $\lceil n / 2\rceil$ positions and the columns in $C$ at the last $\lceil n / 2\rceil$ positions. The key element for the improvement is that no specific order is required for the rows in $R$ nor for the columns in $C$. The algorithm rejects the matrix $A$ for the subsets $R$ and $C$ if $\omega(A[R, C])>1$, otherwise we can write $P A Q$ as in Figure 2(a), where $A_{1}$ and $A_{2}$ are matrices of order $\lceil n / 2\rceil=n / 2$, and we proceed to recursively check that both $A_{1}$ and $A_{2}$ are pet matrices.

The case $n$ is odd is a little bit more complicated. First, the algorithm rejects matrix $A$ for the subsets $R$ and $C$ if $\omega(A[R, C])>1$. Otherwise, we need to consider two (possibly positive) cases: (i) $\omega(A[R, C])=1$ or (ii) $\omega(A[R, C])=0$. If $\omega(A[R, C])=1$, we let $P$ and $Q$ be two permutation matrices that put the rows in $R$ at the first $\lceil n / 2\rceil$ positions and the columns of $C$ at the last $\lceil n / 2\rceil$ positions (no specific order for the rows in $R$ nor for the columns in $C$, except that the 1 of $A$ is at row index $\lceil n / 2\rceil$ and at column index $\lceil n / 2\rceil$ in $P A Q)$. We can write $P A Q$ as in Figure 2(b), where $A_{1}$ and $A_{2}$ are matrices of order $\lceil n / 2\rceil-1=n / 2$, and we proceed to recursively check that both $A_{1}$ and $A_{2}$ are pet matrices. Finally, if $\omega(A[R, C])=0$, for every row index $i \in R$ and every column index $j \in C$, we let $P$ and $Q$ be two permutation matrices that put the rows in $R$ at the first $\lceil n / 2\rceil$ positions and the columns of $C$ at the last $\lceil n / 2\rceil$ positions (no specific order for the rows in $R$ nor for the columns in $C$ except that row $i$ in $A$ is at row index $\lceil n / 2\rceil$ and column index $j$ is at column index $\lceil n / 2\rceil$ in $P A Q)$. We can write $P A Q$ as in Figure 2(c), where $A_{1}$ and $A_{2}$ are matrices of order $\lceil n / 2\rceil-1=n / 2$, and we proceed to recursively check that both $A_{1}$ and $A_{2}$ are pet matrices.

A detailed description is given in Algorithms 1, 2 and 3. We now turn to evaluating the time complexity of this algorithm and we write $T(n)$ for the time complexity of calling perm $\operatorname{Triangular}(A)$ for some $(0,1)$-matrix $A$ or order $n$.

$$
T(n) \leq \begin{cases}(\lceil n / 2\rceil)^{2}\left(\begin{array}{c}
n \\
\lceil n / 2\rceil
\end{array}\right)^{2}(2 T(\lfloor n / 2\rfloor)+1)+O\left(n^{3} \sqrt{n}\right) & \text { if } n \text { is odd } \\
2(\lceil n / 2\rceil)^{2}\left(\begin{array}{c}
n \\
\lceil n / 2\rceil
\end{array}\right)^{2} T(\lfloor n / 2\rfloor)+O\left(n^{3} \sqrt{n}\right) & \text { if } n \text { is odd }\end{cases}
$$

with $T(1)=O(1)$. The $O\left(n^{3} \sqrt{n}\right)$ term is the time complexity for lines 2 and 3 in Algorithm 1. We also observe that the worst case occurs when $n=2^{m}-1$ as $\lceil n / 2\rceil,\lceil n / 4\rceil, \ldots$ are odd integers. Looking for an asymptotic solution of the worst case, we thus write the following simplified recurrence: $T\left(2^{m}\right)=$ 

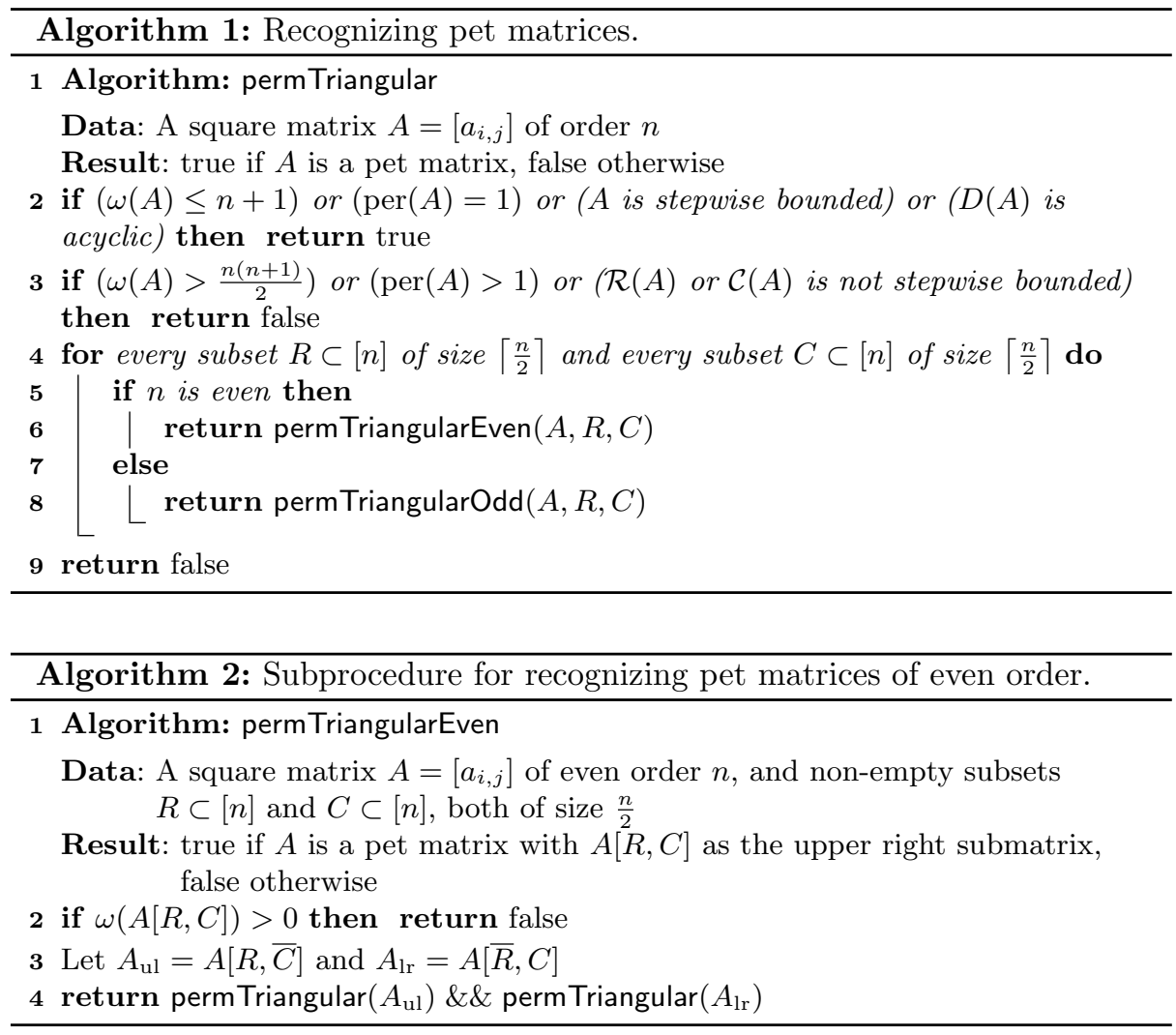

$2^{2 m-2}\left(\begin{array}{c}2^{m} \\ 2^{m-1}\end{array}\right)^{2}\left(2 T\left(2^{m-1}\right)+1\right)+2^{7 m / 6}$, with $T(1)=1$. Now, write $\alpha\left(2^{m}\right)=$ $2^{2 m-2}\left(\begin{array}{c}2^{m} \\ 2^{m-1}\end{array}\right)^{2}$. Clearly, $\alpha\left(2^{m}\right) \geq 2^{7 m / 6}$, and hence we focus for now on on the recurrence $T\left(2^{m}\right)=2 \alpha\left(2^{m}\right)\left(T\left(2^{m-1}\right)+1\right)$. A convenient non-recursive form of $T\left(2^{m}\right)$ is given in the following lemma.

Lemma 7. $T\left(2^{m}\right)=\left(2^{m} \prod_{i=1}^{m} \alpha\left(2^{i}\right)\right)+\left(\sum_{i=1}^{m} 2^{m-i+1} \prod_{j=i}^{m} \alpha\left(2^{j}\right)\right)$.

We now need the following lemma, in order to give an asymptotic solution for $T(n)$ in Proposition 1.

Lemma 8. $\sum_{i=1}^{m} 2^{m-i} \prod_{j=i}^{m} \alpha\left(2^{j}\right)=O\left(m 2^{2^{m+2}+m+1}\right)$.

Proposition 1. Algorithm perm Triangular runs in $O\left(n 2^{4 n} \pi^{-\log (n)}\right)$ time.

Proof. We have already observed that the worst case occurs for $n=2^{m}-1$. According to Lemma 8, we have $T\left(2^{m}\right)=O\left(2^{2^{m+2}+m-3} \pi^{-m}\right)$ and hence $T(n)=O\left(2^{2^{\log (n)+2}+\log (n)-3} \pi^{-\log (n)}\right)=O\left(n 2^{4 n} \pi^{-\log (n)}\right)$. 


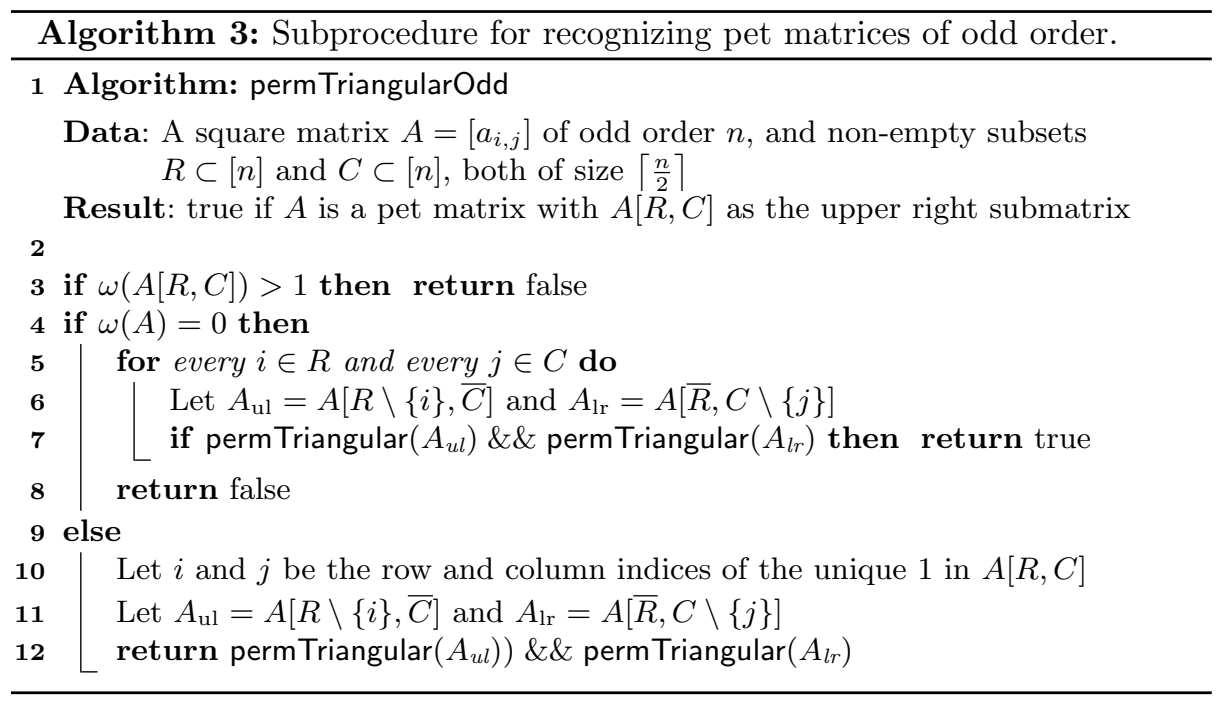

\section{Conclusion}

We suggest for further research directions regarding the hardness of recognizing pet $(0,1)$-matrices. (i) Suppose a $(0,1)$-matrix $A$ of order $n$ has $n+k 1$ 's with $n+2 \leq n+k \leq \frac{1}{2} n(n+1)$. Can one decide in $f(k) n^{O(1)}$ time whether $A$ is a pet matrix, where $f$ is an arbitrary function depending only on $k$ ? (ii) What is the average running time of Algorithm perm Triangular for pet matrices? (iii) A graph labeling strongly related to symmetric pet $(0,1)$-matrices can be defined as follows: Given a graph $G=(V, E)$ or order $n$, decide whether there exists a bijective mapping $f: V \rightarrow[n]$ such that $f(u)+f(v)>n$ for every edge $\{u, v\} \in E$ (i.e., $P A P^{T} \leq \Delta_{n}$ ). Investigating the relationships between the two combinatorial problems is expected to yield fruitful results.

\section{References}

1. R.A. Brualdi and H.J. Ryser. Combinatorial Matrix Theory. Cambridge University Press, New York, 1991.

2. S.A. Cook. The complexity of theorem-proving procedures. In Proc. 3rd Annual ACM Symposium on Theory of Comput., pages 151-158, New York, 1971. ACM.

3. B. DasGupta, T. Jiang, S. Kannan, M. Li, and E. Sweedyk. On the complexity and approximation of syntenic distance. Discrete Applied Mathematics, 88(1-3):59-82, 1998.

4. G.H. Golub and C.F. Van Loan. Matrix Computations. Johns Hopkins University Press, Baltimore and London, 3rd edition, 1996.

5. R.L. Graham, D.E. Knuth, and O. Patashnik. Concrete Mathematics. AddisonWesley, second edition, 1994.

6. J.E. Hopcroft and R.M. Karp. An $O\left(n^{2.5}\right)$ algorithm for matching in bipartite graphs. SIAM Journal on Computing, 4:225-231, 1975. 
7. H.S. Wilf. On crossing numbers, and some unsolved problems. In B. Bollobás and A. Thomason, editors, Combinatorics, Geometry and Probability: A Tribute to Paul Erdös, pages 557-562. Cambridge University Press, 1997. 


\section{Appendix (Reviewers' version only)}

Proof (of Lemma 1). The forward direction if obvious. For the reverse direction, let $\mathcal{S}=\left(S_{i}: 1 \leq i \leq n\right)$ be a configuration of subsets of some ground set of cardinality $n$ and $\varphi: \mathcal{S} \rightarrow[n]$ be a bijective mapping such that $\left|\bigcup_{S \in \mathcal{S}: \varphi(S) \leq i} S\right| \leq$ $i$ for all $1 \leq i \leq n$. Let $A=\left[a_{i, j}\right]$ be the incidence matrix of $\mathcal{S}$. Now let $P$ be the permutation matrix of order $n$ that permutes the rows of $A$ so that row $i$ of $P A$ correspond to subset $\varphi^{-1}(i)$ for all $1 \leq i \leq n$. We denote this row-permuted matrix by $A^{\prime}=\left[a_{i, j}^{\prime}\right]$. Define the function $\tau:[n] \rightarrow[n+1]$ that indicates for each column index $j$ the minimum row index $i$ so that $a_{i, j}^{\prime}=1$, and we adhere to the convention that $\tau(j)=n+1$ if row $j$ does not contain a 1 . Define a permutation matrix $Q$ or order $n$ that permutes the columns of $A^{\prime}$ by ascending $\tau$ values, breaking ties arbitrarily. We designate this row-permuted matrix by $A^{\prime \prime}=\left[a_{i, j}^{\prime \prime}\right]$. We claim that $A^{\prime \prime}=P A Q \leq \triangle_{n}$. Indeed, suppose, aiming at a contradiction, that $A^{\prime \prime} \not \leq \triangle_{n}$. Let $i_{\min }$ be the smallest row index such that $a_{i_{\min }, j_{\min }}^{\prime \prime}=1$ for some $j_{\min }>i_{\min }$. Then it follows that $\tau(j) \leq \tau\left(j_{\min }\right)$ for $j<j_{\min }$ and hence $\left|\bigcup_{S \in \mathcal{S}: \varphi(S) \leq i_{\min }} S\right|>i_{\min }$. This is the desired contradiction, and hence $A^{\prime \prime}=P A Q \leq \triangle_{n}$.

Proof (of Lemma 2). The proof is by contradiction. Denote by $\Phi(\mathcal{S})$ the set of all sbl s of the configuration of subsets $\mathcal{S}$. We claim that there exists a normalized sbl $\varphi \in \Phi(\mathcal{S})$ which maps the identical subsets of $\mathcal{S}$ to sets of consecutive integers. For each $\varphi \in \Phi(\mathcal{S})$, define

$$
\mathcal{M}(\varphi)=\left\{S_{j} \mid \exists S_{i}, S_{k} \text { s.t. } \varphi\left(S_{i}\right)<\varphi\left(S_{k}\right)<\varphi\left(S_{j}\right) \text { and } S_{i}=S_{j} \neq S_{k}\right\}
$$

Then, there exists a mapping $\varphi^{*} \in \Phi(\mathcal{S})$ such that $\left|\mathcal{M}\left(\varphi^{*}\right)\right| \leq\left|\mathcal{M}\left(\varphi^{\prime}\right)\right|$ for all $\varphi^{\prime} \in \Phi(\mathcal{S})$. We show that $\left|\mathcal{M}\left(\varphi^{*}\right)\right|=0$, and hence that $\varphi^{*}$ is our desired mapping. Suppose, for the sake of contradiction, that $\left|\mathcal{M}\left(\varphi^{*}\right)\right|>0$. Let $S_{j} \in \mathcal{M}\left(\varphi^{*}\right)$ be such that $\varphi^{*}\left(S_{j}\right) \leq \varphi^{*}(S)$ for all $S \in \mathcal{M}\left(\varphi^{*}\right)$. Then, there exists two subsets $S_{i}, S_{k} \in \mathcal{S}$ such that $S_{i}=S_{j} \neq S_{k}$ and $\varphi^{*}\left(S_{i}\right)+1=\varphi^{*}\left(S_{k}\right)<\varphi^{*}\left(S_{j}\right)$. Consider a new labeling $\varphi^{\prime}$ defined by

$$
\begin{array}{ll}
\varphi^{\prime}(S)=\varphi^{*}(S) & \text { for all } S \in \mathcal{S} \text { such that } 1 \leq \varphi^{*}(S) \leq \varphi^{*}\left(S_{i}\right) \\
\varphi^{\prime}(S)=\varphi^{*}(S)+1 & \text { for all } S \in \mathcal{S} \text { such that } \varphi^{*}\left(S_{i}\right)<\varphi^{*}(S)<\varphi^{*}\left(S_{j}\right) \\
\varphi^{\prime}\left(S_{j}\right)=\varphi^{*}\left(S_{k}\right) & \\
\varphi^{\prime}(S)=\varphi^{*}(S)+1 & \text { for all } S \in \mathcal{S} \text { such that } \varphi^{*}\left(S_{j}\right)<\varphi^{*}(S) \leq m
\end{array}
$$

For simplicity of notation, we write $i$ instead of $\varphi^{*}\left(S_{i}\right), j$ instead of $\varphi^{*}\left(S_{j}\right)$ and $k$ instead of $\varphi^{*}\left(S_{k}\right)$. Observe that $i+1=k$. We claim that $\varphi^{\prime}$ is an sbl for $\mathcal{S}$. It is sufficient to show that

$$
\bigcup_{\varphi^{\prime}(S) \leq \ell} S \subseteq \bigcup_{\varphi^{*}(S) \leq \ell} S
$$


for all $k \leq \ell \leq j$. We check at once that

$$
\begin{aligned}
\bigcup_{1 \leq \varphi^{\prime}(S) \leq \ell} S & =\left(\bigcup_{1 \leq \varphi^{\prime}(S) \leq i} S\right) \cup S_{j} \cup\left(\bigcup_{k+1 \leq \varphi^{\prime}(S) \leq \ell} S\right) \\
& =\left(\bigcup_{1 \leq \varphi^{*}(S) \leq i} S\right) \cup S_{j} \cup\left(\bigcup_{k \leq \varphi^{*}(S) \leq \ell-1} S\right) \\
& =\left(\bigcup_{1 \leq \varphi^{*}(S) \leq \ell-1} S\right) \cup S_{j} \\
& =\bigcup_{1 \leq \varphi^{*}(S) \leq \ell-1} S \\
& \subseteq \underset{1 \leq \varphi^{*}(S) \leq \ell}{\bigcup} S
\end{aligned}
$$

It follows immediately that $S_{j} \notin \mathcal{M}\left(\varphi^{\prime}\right)$. Indeed, $\varphi^{*}\left(S_{j}\right) \leq \varphi^{*}(S)$ for all $S \in$ $\mathcal{M}\left(\varphi^{*}\right)$.

We proceed to show that $\left|\mathcal{M}\left(\varphi^{*}\right)\right|>\left|\mathcal{M}\left(\varphi^{\prime}\right)\right|$. If $\left|\mathcal{M}\left(\varphi^{\prime}\right)\right|=0$, we are done, so that we may assume $\left|\mathcal{M}\left(\varphi^{\prime}\right)\right|>0$. Let $S^{\prime} \in \mathcal{M}\left(\varphi^{\prime}\right)$. Then there exist subsets $S_{a}$ and $S_{b}$ such that $\varphi^{\prime}\left(S_{a}\right)<\varphi^{\prime}\left(S_{b}\right)<\varphi^{\prime}\left(S^{\prime}\right)$ and $S_{a}=S^{\prime} \neq S_{b}$. Now, observe that we must have $\varphi^{*}\left(S_{j}\right)<\varphi^{*}\left(S^{\prime}\right)$. Again, this follows from the fact that $\varphi^{*}\left(S_{j}\right) \leq \varphi^{*}(S)$ for all $S \in \mathcal{M}\left(\varphi^{*}\right)$. Therefore, by construction of the labeling $\varphi^{\prime}$, either $\varphi^{*}\left(S_{a}\right)<\varphi^{*}\left(S_{b}\right)<\varphi^{*}\left(S^{\prime}\right)$ or $\varphi^{*}\left(S_{b}\right)<\varphi^{*}\left(S_{a}\right)<\varphi^{*}\left(S^{\prime}\right)$. We claim that $S^{\prime} \in \mathcal{M}\left(\varphi^{*}\right)$. The result is certainly valid in case $\varphi^{*}\left(S_{a}\right)<\varphi^{*}\left(S_{b}\right)$. Suppose now that $\varphi^{*}\left(S_{b}\right)<\varphi^{*}\left(S_{a}\right)$. Then it follows that we must have $S_{a}=S_{j}$. But $S_{i}=S_{j}$. Therefore $\varphi^{*}\left(S_{i}\right)<\varphi^{*}\left(S_{b}\right)<\varphi^{*}\left(S^{\prime}\right)$ and $S_{i}=S^{\prime} \neq S_{b}$, and hence $S^{\prime} \in \mathcal{M}\left(\varphi^{*}\right)$. Combining this with the fact that $S_{j} \notin \mathcal{M}\left(\varphi^{\prime}\right)$ yields $\left|\mathcal{M}\left(\varphi^{*}\right)\right|>\left|\mathcal{M}\left(\varphi^{\prime}\right)\right|$. This contradicts the choice of $\varphi^{*}$. Therefore, we must have $\left|\mathcal{M}\left(\varphi^{*}\right)\right|=0$ and hence $\varphi^{*}$ is a normalized sbl of $\mathcal{S}$.

Proof (of Lemma 3). We claim that there exists a satisfying truth assignment $f$ for $\phi$ if and only if there exists an sbl of the configuration of subsets $C$ of the ground set $\mathbf{X}$.

Suppose that there exists a satisfying truth assignment $f$ for the $3 \mathrm{CNF}$ formula $\phi$. Due to symmetry, there is no loss of generality in assuming that each clause is satisfied by its first literal. Define an labeling $\tau$ of the configuration of subsets $C$ as follows. First, we begin our labeling construction by placing all the empty subsets of $C$, that is

$$
1 \leq \tau\left(\mathcal{B}_{i}\right) \leq p_{1}-1
$$


for all $\mathcal{B}_{i} \in B$. Next, all the subsets corresponding to the variables are ordered as follows: for all $\mathcal{V}_{i, j} \in \mathcal{V}_{i}, 1 \leq i \leq n$,

$$
p_{i}+\sum_{k=1}^{i-1}\left(p_{k}+q_{k}+r_{k}\right) \leq \tau\left(\mathcal{V}_{i, j}\right) \leq p_{i}+q_{i}+\sum_{k=1}^{i-1}\left(p_{k}+q_{k}+r_{k}\right)-1
$$

The satisfying truth assignment $f$ for $\phi$ is coded in our construction as follows: for all $\mathcal{A}_{i, j} \in \mathcal{A}_{i}, 1 \leq i \leq n$, where $\mathcal{A}_{i}=\mathcal{T}_{i}$ if $f\left(x_{i}\right)=$ TRUE and $\mathcal{A}_{i}=\mathcal{F}_{i}$ if $f\left(x_{i}\right)=$ FALSE

$$
p_{i}+q_{i}+\sum_{k=1}^{i-1}\left(p_{k}+q_{k}+r_{k}\right) \leq \tau\left(\mathcal{A}_{i, j}\right) \leq \sum_{k=1}^{i}\left(p_{k}+q_{k}+r_{k}\right)-1
$$

and for all $\mathcal{A}_{i, j}^{\prime} \in \mathcal{A}_{i}^{\prime}, 1 \leq i \leq n$, where $\mathcal{A}_{i}^{\prime}=\mathcal{T}_{i}^{\prime}$ if $f\left(x_{i}\right)=$ TRUE and $\mathcal{A}_{i}^{\prime}=\mathcal{F}_{i}^{\prime}$ if $f\left(x_{i}\right)=$ FALSE,

$$
\sum_{k=1}^{i}\left(p_{k}+q_{k}+r_{k}\right) \leq \tau\left(\mathcal{A}_{i, j}^{\prime}\right) \leq p_{i+1}+\sum_{k=1}^{i}\left(p_{k}+q_{k}+r_{k}\right)-1
$$

It easily follows that

$$
\tau\left(\mathcal{V}_{i}\right)<\tau\left(\mathcal{A}_{i}\right)<\tau\left(\mathcal{A}_{i}^{\prime}\right)<\tau\left(\mathcal{V}_{j}\right)<\tau\left(\mathcal{A}_{j}\right)<\tau\left(\mathcal{A}_{j}^{\prime}\right)
$$

for all $1 \leq i<j \leq n$.

Having disposed of all those subsets corresponding to the variables and to the satisfying truth assignment $f$ for $\phi$, we now turn to the clauses. This will be divided into two parts. First, the subsets of $\mathcal{C}_{i}, 1 \leq i \leq m$, are ordered as follows: for all $\mathcal{C}_{i, j} \in \mathcal{C}_{i}, 1 \leq i \leq m$,

$$
p_{n+i}+\sum_{k=1}^{n+i-1}\left(p_{k}+q_{k}+r_{k}\right) \leq \tau\left(\mathcal{C}_{i, j}\right) \leq p_{n+i}+q_{n+i}+\sum_{k=1}^{n+i-1}\left(p_{k}+q_{k}+r_{k}\right)-1
$$

Second, all the subsets of $\mathcal{L}_{i, 1}$ and $\mathcal{L}_{i, 1}^{\prime}, 1 \leq i \leq m$, are ordered as follows ${ }^{1}$ : for all $\mathcal{L}_{i, 1, j} \in \mathcal{L}_{i, 1}, 1 \leq i \leq m$,

$$
p_{n+i}+q_{n+i}+\sum_{k=1}^{n+i-1}\left(p_{k}+q_{k}+r_{k}\right) \leq \tau\left(\mathcal{L}_{i, 1, j}\right) \leq \sum_{k=1}^{n+i}\left(p_{k}+q_{k}+r_{k}\right)-1
$$

and for all $\mathcal{L}_{i, 1, j}^{\prime} \in \mathcal{L}_{i, 1}^{\prime}, 1 \leq i \leq m$,

$$
\sum_{k=1}^{n+i}\left(p_{k}+q_{k}+r_{k}\right) \leq \tau\left(\mathcal{L}_{i, 1, j}^{\prime}\right) \leq p_{n+i+1}+\sum_{k=1}^{n+i}\left(p_{k}+q_{k}+r_{k}\right)-1
$$

\footnotetext{
${ }^{1}$ Recall that we assume that each clause is satisfied by its first literal.
} 
A trivial verification shows that

$$
\tau\left(\mathcal{C}_{i}\right)<\tau\left(\mathcal{L}_{i, 1}\right)<\tau\left(\mathcal{L}_{i, 1}^{\prime}\right)<\tau\left(\mathcal{C}_{j}\right)<\tau\left(\mathcal{L}_{j, 1}\right)<\tau\left(\mathcal{L}_{j, 1}^{\prime}\right)
$$

for all $1 \leq i<j \leq m$.

Here come the $K$ separator subsets of $\mathcal{S}$, that is for all $\mathcal{S}_{i} \in \mathcal{S}$

$$
p_{n+m+1}+\sum_{k=1}^{n+m}\left(p_{k}+q_{k}+r_{k}\right) \leq \tau\left(\mathcal{S}_{i}\right) \leq p_{n+m+1}+K+\sum_{k=1}^{n+m}\left(p_{k}+q_{k}+r_{k}\right)-1
$$

Up to now, all the subsets of $C$ but $L$ of them have been used to define $\tau$. Our labeling construction ends with those subsets by packing them in the $L$ last places:

$p_{n+m+1}+K+\sum_{k=1}^{n+m}\left(p_{k}+q_{k}+r_{k}\right) \leq \tau\left(\mathcal{X}_{j}\right) \leq p_{n+m+1}+K+L+\sum_{k=1}^{n+m}\left(p_{k}+q_{k}+r_{k}\right)-1$

A careful examination of $\tau$ shows that $\left|\bigcup_{\tau(X) \leq i} X\right| \leq i$ for $1 \leq i \leq|C|$ and hence that $\tau$ is an $\mathrm{sbl}^{2}$ of the configuration of subsets $C$ of the ground set $\mathbf{X}$. An (partial) illustration of the construction of the sbl $\tau$ is shown in Figure 1.

For the converse, suppose that there exists an $\operatorname{sbl} \tau$ for $C$, that is an labeling such that $\left|\bigcup_{\tau\left(X_{j}\right) \leq i} X_{j}\right| \leq i$ for $1 \leq i \leq|C|$. According to lemma 2 , there is no loss of generality in assuming that $\tau$ is a normalized sbl i.e., the identical subsets of the configuration $C$ are mapped by $\tau$ to a set of consecutive integers. This property is crucial in our proof.

Let us start by proving that $1 \leq \tau\left(\mathcal{B}_{i}\right) \leq p_{1}-1$ for all $\mathcal{B}_{i} \in \mathcal{B}$. Indeed, as mentioned earlier in the proof, all the above defined subsets of the configuration of subsets $C$ but those of $\mathcal{B}$ contain at least $p_{1}$ elements and hence can not be mapped to an integer $i \leq p_{1}-1$ in any $\operatorname{sbl} \tau$ for $C$.

Define the subcollection $C^{*} \subset C$ as follows:

$$
C^{*}=\left(X \in C \mid \max \left\{\tau\left(\mathcal{B}_{i}\right) \mid \mathcal{B}_{i} \in \mathcal{B}\right\}<\tau(X)<\min \left\{\tau\left(\mathcal{S}_{i}\right) \mid \mathcal{S}_{i} \in \mathcal{S}\right\}\right)
$$

In other words, the subcollection $C^{*}$ contains all those subsets that go after the last bootstrap subset $\mathcal{B}_{i} \in \mathcal{B}$ and before the first separator subset $\mathcal{S}_{i} \in \mathcal{S}$. We claim that $C^{*}$ contains (1) the subsets of $\mathcal{V}_{i}$ for $1 \leq i \leq n,(2)$ either the subsets of $\mathcal{T}_{i}$ and $\mathcal{T}_{i}^{\prime}$ or the subsets of $\mathcal{F}_{i}$ and $\mathcal{F}_{i}^{\prime}$ for $1 \leq i \leq n,(3)$ the subsets of $\mathcal{C}_{i}$ for $1 \leq i \leq m$ and (4) the subsets of $\mathcal{L}_{i, 1}$ and $\mathcal{L}_{i, 1}^{\prime}$ or the subsets of $\mathcal{L}_{i, 2}$ and $\mathcal{L}_{i, 2}^{\prime}$ or the subsets of $\mathcal{L}_{i, 3}$ and $\mathcal{L}_{i, 3}^{\prime}$ for $1 \leq i \leq m$. For simplicity, this will be divided into several steps.

Having disposed of the bootstrap subsets, we now turn to the non-empty subsets of the configuration of subsets $C$. Roughly speaking, we show that our

\footnotetext{
${ }^{2}$ Observe that the construction of $\tau$ may be specialized to yield a normalized sbl. Indeed, all the subsets of $C$ but the $L$ last ones are mapped by $\tau$ to a set of consecutive integers. But there is no less of generality in assuming that these subsets are mapped by $\tau$ to a set of consecutive integers as well.
} 
construction implies a force placement of the subsets of $C$ together with choices corresponding to a satisfying truth assignment for the $3 \mathrm{CNF}$ formula $\phi$. First, we must have:

$$
p_{1} \leq \tau\left(\mathcal{V}_{1, j}\right) \leq p_{1}+q_{1}-1
$$

for all $1 \leq j \leq q_{1}$. Indeed, all other subsets have more than $p_{1}$ elements and hence can not be mapped to $p_{1}$ by $\tau$. Moreover, $\tau$ is a normalized $\operatorname{sbl}$ for $C$, i.e., the subsets $\mathcal{V}_{1, j}, 1 \leq j \leq q_{1}$, are mapped by $\tau$ to a set of consecutive integers. Now, the key point is that the subset which is mapped to $p_{1}+q_{1}$ by $\tau$ must have cardinality at most $p_{1}+q_{1}$. But a careful examination of the configuration of subsets $C$ shows that all the remaining subsets contain $\mathbf{V}_{1}$, and hence the subset which is mapped to $p_{1}+q_{1}$ by $\tau$ may introduce at most $q_{1}$ new elements of the ground set. Then it follows from our construction that this subset is either a subset of the collection $\mathcal{T}_{1}$ or a subset of the collection $\mathcal{F}_{1}$. Indeed, it is sufficient to note that $r_{1}+p_{2}>q_{1}$, and hence that no subset $\mathcal{V}_{2, j} \in \mathcal{V}_{2}$ can satisfy $\tau\left(\mathcal{V}_{2, j}\right)=p_{1}+q_{1}$. As an immediate result, exactly one of the following two statements is true:

$$
\begin{aligned}
& p_{1}+q_{1} \leq \tau\left(\mathcal{T}_{1, j}\right) \leq p_{1}+q_{1}+r_{1}-1 \\
& p_{1}+q_{1} \leq \tau\left(\mathcal{F}_{1, j}\right) \leq p_{1}+q_{1}+r_{1}-1
\end{aligned}
$$

for all $1 \leq j \leq r_{1}$. We can now proceed analogously to the above to obtain:

$$
\begin{aligned}
& (1) \quad \Rightarrow \quad p_{1}+q_{1}+r_{1} \leq \tau\left(\mathcal{T}_{1, j}^{\prime}\right) \leq p_{1}+q_{1}+r_{1}+p_{2}-1 \\
& (2) \quad \Rightarrow \quad p_{1}+q_{1}+r_{1} \leq \tau\left(\mathcal{F}_{1, j}^{\prime}\right) \leq p_{1}+q_{1}+r_{1}+p_{2}-1
\end{aligned}
$$

for all $1 \leq j \leq p_{2}$. Summarizing, exactly one of the following two statements is true:

$$
\begin{aligned}
p_{1} \leq \tau\left(\mathcal{V}_{1}\right)<\tau\left(\mathcal{T}_{1}\right)<\tau\left(\mathcal{T}_{1}^{\prime}\right) & \leq p_{1}+q_{1}+r_{1}+p_{2}-1 \\
p_{1} \leq \tau\left(\mathcal{V}_{1}\right)<\tau\left(\mathcal{F}_{1}\right) & <\tau\left(\mathcal{F}_{1}^{\prime}\right) \leq p_{1}+q_{1}+r_{1}+p_{2}-1
\end{aligned}
$$

We continue in this fashion obtaining that exactly one of the following two statements is true:

$$
\begin{aligned}
& p_{i}+\sum_{k=1}^{i-1}\left(p_{k}+q_{k}+r_{k}\right) \leq \tau\left(\mathcal{V}_{i}\right)<\tau\left(\mathcal{T}_{i}\right)<\tau\left(\mathcal{T}_{i}^{\prime}\right) \leq p_{i+1}+\sum_{k=1}^{i}\left(p_{k}+q_{k}+r_{k}\right)-1 \\
& p_{i}+\sum_{k=1}^{i-1}\left(p_{k}+q_{k}+r_{k}\right) \leq \tau\left(\mathcal{V}_{i}\right)<\tau\left(\mathcal{F}_{i}\right)<\tau\left(\mathcal{F}_{i}^{\prime}\right) \leq p_{i+1}+\sum_{k=1}^{i}\left(p_{k}+q_{k}+r_{k}\right)-1
\end{aligned}
$$

for all $1 \leq i \leq n$. This result is crucial as it allows us to construct a truth assignment $f$ for the $3 \mathrm{CNF}$ formula $\phi$. Indeed, subsets of $\mathcal{T}_{i}$ and $\mathcal{T}_{i}^{\prime}$ are associated in our construction to literal $x_{i}$ while subsets of $\mathcal{F}_{i}$ and $\mathcal{F}_{i}^{\prime}$ are associated in our construction to literal $\bar{x}_{i}$. 
Having disposed of the variables we now turn to the clauses. For the sake of clarity, let us first introduce the temporary notations

$$
\begin{aligned}
l_{i} & =p_{n+i}+\sum_{k=1}^{n+i-1}\left(p_{k}+q_{k}+r_{k}\right) \\
h_{i} & =p_{n+i+1}+\sum_{k=1}^{n+i}\left(p_{k}+q_{k}+r_{k}\right)-1
\end{aligned}
$$

for $1 \leq i \leq m$. We may now proceed analogously to the above to obtain that exactly one of the following three statements is true:

$$
\begin{aligned}
& l_{i} \leq \tau\left(\mathcal{C}_{i}\right)<\tau\left(\mathcal{L}_{i, 1}\right)<\tau\left(\mathcal{L}_{i, 1}^{\prime}\right) \leq h_{i} \\
& l_{i} \leq \tau\left(\mathcal{C}_{i}\right)<\tau\left(\mathcal{L}_{i, 2}\right)<\tau\left(\mathcal{L}_{i, 2}^{\prime}\right) \leq h_{i} \\
& l_{i} \leq \tau\left(\mathcal{C}_{i}\right)<\tau\left(\mathcal{L}_{i, 3}\right)<\tau\left(\mathcal{L}_{i, 3}^{\prime}\right) \leq h_{i}
\end{aligned}
$$

for all $1 \leq i \leq m$.

According to the above, the subcollection $C^{*}$ contains either the subsets of $\mathcal{T}_{i}$ and $\mathcal{T}_{i}^{\prime}$ or the subsets of $\mathcal{F}_{i}$ and $\mathcal{F}_{i}^{\prime}$ for $1 \leq i \leq n$. Therefore we can define a truth assignment $f$ for the 3CNF formula $\phi$ as follows: $f\left(x_{i}\right)=$ TRUE if $\mathcal{T}_{i} \subset C^{*}$ and $f\left(x_{i}\right)=$ FALSE if $\mathcal{F}_{i} \subset C^{*}$ for $1 \leq i \leq n$. We claim that $f$ is a satisfying truth assignment for $\phi$. Indeed, for each $i, 1 \leq i \leq m$, consider the true statement

$$
l_{i} \leq \tau\left(\mathcal{C}_{i}\right)<\tau\left(\mathcal{L}_{i, k}\right)<\tau\left(\mathcal{L}_{i, k}^{\prime}\right) \leq h_{i}
$$

where $k$ is either 1,2 or 3 . By construction we have

$$
\mathcal{L}_{i, k, j}=\mathbf{V} \cup \mathbf{V}^{\prime} \cup \mathbf{A}_{k} \cup \mathbf{L}_{i, k} \cup\left(\bigcup_{1 \leq \ell \leq i} \mathbf{C}_{\ell}\right) \cup\left(\bigcup_{1 \leq \ell \leq i-1} \mathbf{C}_{\ell}^{\prime}\right)
$$

for $1 \leq j \leq r_{n+i}$ and

$$
\mathcal{L}_{i, k, j}^{\prime}=\mathbf{V} \cup \mathbf{V}^{\prime} \cup \mathbf{A}_{k} \cup \mathbf{L}_{i, k} \cup\left(\bigcup_{1 \leq \ell \leq i} \mathbf{C}_{\ell}\right) \cup\left(\bigcup_{1 \leq \ell \leq i} \mathbf{C}_{\ell}^{\prime}\right)
$$

for $1 \leq j \leq p_{n+i+1}$, where $\mathbf{A}_{k}=\mathbf{T}_{\ell}$ if $\lambda_{i, k}=x_{\ell}$ and $\mathbf{A}_{k}=\mathbf{F}_{\ell}$ if $\lambda_{i, k}=\bar{x}_{\ell}$. But a careful examination of the configuration of subsets $C$ and of the sbl $\tau$ shows that none of the previous subsets contain the elements of $\mathbf{L}_{i, k}$. Since $\left|\mathcal{C}_{i}\right|=q_{n+i}$ and $\left|\mathbf{L}_{i, k}\right|=q_{n+i}$, then it follows that all the elements of $\mathbf{A}_{k}$ must have been introduced by previous subsets. Indeed, $l_{i} \leq \tau\left(\mathcal{C}_{i}\right)<\tau\left(\mathcal{L}_{i, k}\right)<\tau\left(\mathcal{L}_{i, k}^{\prime}\right) \leq h_{i}$ and hence $\mathcal{C}_{i}$ and $\mathcal{L}_{i, k}$ are mapped by $\tau$ to a set of consecutive integers. Therefore, if $\mathbf{A}_{k}=\mathbf{T}_{\ell}$ (resp. $\mathbf{A}_{k}=\mathbf{F}_{\ell}$ ) then we must have chosen $\mathcal{T}_{\ell}$ (resp. $\mathcal{F}_{\ell}$ ) in a previous step, that is $\mathcal{T}_{\ell}, \mathcal{T}_{\ell}^{\prime} \in C^{*}$ (resp. $\mathcal{F}_{\ell}, \mathcal{F}_{\ell}^{\prime} \in C^{*}$ ). Then it follows that clause $c_{i}$ is satisfied by its $k$-th literal. Hence, $f$ is a satisfying truth assignment for $\phi$ and the lemma is proved. 
Proof (of Lemma 4). The proof is by induction. The result is obvious for $n=1$. For the inductive step, assume the statement holds for some natural number $n$ and let $A$ be a $(0,1)$-matrix of order $n+1$. Since $A$ is assumed to be a pet matrix there exist permutation matrices $P$ and $Q$ such that $P A Q \leq \triangle_{n}$. Let $A^{\prime}=P A Q$. As $\operatorname{per}(A)$ remains invariant under arbitrary permutation of the lines of $A$ we have $\operatorname{per}(A)=\operatorname{per}\left(A^{\prime}\right)$. If $\operatorname{per}\left(A^{\prime}\right)=0$, we are done. Otherwise $\operatorname{per}\left(A^{\prime}\right) \geq 1$ and hence $n 1^{\prime}$ 's appear on the main diagonal of $A^{\prime}$. We designate the matrix obtained from $A^{\prime}$ by deleting the first row and the first column permuted matrix by $A^{\prime \prime}$. As the first row of $A^{\prime}$ contains exactly one 1 , we have $\operatorname{per}\left(A^{\prime \prime}\right)=\operatorname{per}\left(A^{\prime}\right)=\operatorname{per}(A) \leq 1$, thereby proving the lemma.

Proof (of Lemma 5). The proof is by induction. The assertion is certainly valid for $n=2$ as all $(0,1)$-matrix of order 2 are pet matrices but $J_{2}$. For the inductive step, assume the statement holds for some natural number $n$ and let $A$ be $(0,1)$ matrix of order $n+1$. If $A=\mathbf{0}$ we are done. Otherwise, let $\alpha_{i}$ be a row of $A$ with minimum row sum $r_{i}$. Since $A$ contains at most $n+11$ 's, we certainly have $r_{i} \leq 1$. We need to consider two cases:

- If $r_{i}=0$, let $\beta_{j}$ be a column of $A$ with maximum column sum $c_{j}$. Since $A \neq \mathbf{0}$, we certainly have $c_{j}>0$. We now permute the lines of $A$ so that row $\alpha_{i}$ and column $\beta_{j}$ are the first row and column of the permuted matrix. We now delete the first row and column of this permuted matrix and apply the induction hypothesis to this submatrix of order $n$.

- If $r_{i}=1$, we may permute the rows of $A$ so that row 1 of the permuted matrix contains a 1 in the $(1,1)$ position and $O$ 's elsewhere. We now delete the first row and column of this matrix and apply the induction hypothesis to this submatrix of order $n$.

Proof (of Lemma 7). The proof is by induction on $m$. First, the assertion is certainly valid for $m=1$ as

$$
\begin{aligned}
2 \alpha\left(2^{1}\right)\left(T\left(2^{0}\right)+1\right) & =2 \times 4 \times(1+1) \\
& =16
\end{aligned}
$$

and

$$
\begin{aligned}
\left(2^{1} \prod_{i=1}^{1} \alpha\left(2^{i}\right)\right)+\left(\sum_{i=1}^{1} 2^{1-i+1} \prod_{j=i}^{1} \alpha\left(2^{j}\right)\right) & =\left(2^{1} \times \alpha\left(2^{1}\right)\right)+\left(2^{1} \times \alpha\left(2^{1}\right)\right) \\
& =(2 \times 4)+(2 \times 4) \\
& =16 .
\end{aligned}
$$


Suppose now that the assertion is true for $2^{m}$. Then

$$
\begin{aligned}
T\left(2^{m+1}\right) & =2 \alpha\left(2^{m+1}\right) T\left(2^{m}\right)+2 \alpha\left(2^{m+1}\right) \\
& =2 \alpha\left(2^{m+1}\right)\left(\left(2^{m} \prod_{i=1}^{m} \alpha\left(2^{i}\right)\right)+\left(\sum_{i=1}^{m} 2^{m-i+1} \prod_{j=i}^{m} \alpha\left(2^{j}\right)\right)+2 \alpha\left(2^{m+1}\right)\right. \\
& =\left(2^{m+1} \prod_{i=1}^{m+1} \alpha\left(2^{i}\right)\right)+\left(\sum_{i=1}^{m} 2^{m+1-i+1} \prod_{j=i}^{m+1} \alpha\left(2^{j}\right)\right)+2 \alpha\left(2^{m+1}\right) \\
& =\left(2^{m+1} \prod_{i=1}^{m+1} \alpha\left(2^{i}\right)\right)+\left(\sum_{i=1}^{m+1} 2^{m+1-i+1} \prod_{j=i}^{m+1} \alpha\left(2^{j}\right)\right)-2 \alpha\left(2^{m+1}\right)+2 \alpha\left(2^{m+1}\right) \\
& =\left(2^{m+1} \prod_{i=1}^{m+1} \alpha\left(2^{i}\right)\right)+\left(\sum_{i=1}^{m+1} 2^{m+1-i+1} \prod_{j=i}^{m+1} \alpha\left(2^{j}\right)\right) .
\end{aligned}
$$

Proof (of Lemma 8). We need the following claims.

Claim 1.

$$
\alpha\left(2^{i}\right)=\frac{2^{2^{m+1}+m-1}}{\pi}\left(1+O\left(2^{1-m}\right)\right) .
$$

Proof. We use the following well-known asymptotic (see e.g. [5]):

$$
\left(\begin{array}{c}
2 n \\
n
\end{array}\right)=\frac{4^{n}}{\sqrt{\pi n}}\left(1+O\left(n^{-1}\right)\right) .
$$

Therefore,

$$
\begin{aligned}
\alpha\left(2^{m}\right) & =2^{2 m-2}\left(\begin{array}{c}
2^{m} \\
2^{m-1}
\end{array}\right)^{2} \\
& =2^{2 m-2}\left(\frac{4^{2^{m-1}}}{\sqrt{\pi 2^{m-1}}}\left(1+O\left(2^{1-m}\right)\right)\right)^{2} \\
& =2^{2 m-2}\left(\frac{4^{2^{m-1}}}{\sqrt{\pi 2^{m-1}}}\right)^{2}\left(1+O\left(2^{1-m}\right)\right)^{2} \\
& =2^{2 m-2} \frac{4^{2^{m}}}{\pi 2^{m-1}}\left(1+O\left(2^{1-m}\right)\right)^{2} \\
& =\frac{2^{2^{m+1}+m-1}}{\pi}\left(1+O\left(2^{1-m}\right)\right)^{2} .
\end{aligned}
$$


We now develop the error term to obtain

$$
\begin{aligned}
\alpha\left(2^{m}\right) & =\frac{2^{2^{m+1}+m-1}}{\pi}\left(1+O\left(2^{1-m}\right)\right)\left(1+O\left(2^{1-m}\right)\right) \\
& =\frac{2^{2^{m+1}+m-1}}{\pi}\left(1+O\left(2^{1-m}\right)+O\left(2^{1-m}\right)+O\left(2^{2-2 m}\right)\right) \\
& =\frac{2^{2^{m+1}+m-1}}{\pi}\left(1+O\left(2^{1-m}\right)\right)
\end{aligned}
$$

Claim 2.

$$
\prod_{i=1}^{m} \alpha\left(2^{i}\right)=O\left(\frac{2^{2^{m+2}-3}}{\pi^{m}}\right)
$$

Proof. According to Claim 1, we may write

$$
\begin{aligned}
\prod_{i=1}^{m} \alpha\left(2^{i}\right) & =\prod_{i=1}^{m} \frac{2^{2^{i+1}+i-1}}{\pi}\left(1+O\left(2^{1-i}\right)\right) \\
& =\left(\frac{1}{2 \pi}\right)^{m}\left(\prod_{i=1}^{m} 2^{2^{i+1}}\right)\left(\prod_{i=1}^{m} 2^{i}\right)\left(\prod_{i=1}^{m}\left(1+O\left(2^{1-i}\right)\right)\right) \\
& =\left(\frac{1}{2 \pi}\right)^{m}\left(2^{2^{m+2}-4}\right)\left(2^{m+1}-2\right)\left(\prod_{i=1}^{m}\left(1+O\left(2^{1-i}\right)\right)\right) \\
& =\frac{2^{2^{m+2}-3}}{\pi^{m}}\left(\frac{2^{m}-1}{2^{m}}\right)\left(\prod_{i=1}^{m}\left(1+O\left(2^{1-i}\right)\right)\right) \\
& =\frac{2^{2^{m+2}-3}}{\pi^{m}}\left(1-2^{-m}\right)\left(\prod_{i=1}^{m}\left(1+O\left(2^{1-i}\right)\right)\right) .
\end{aligned}
$$

But $\prod_{i=1}^{m}\left(1+O\left(2^{1-i}\right)\right)=O(1)$ and hence

$$
\begin{aligned}
\prod_{i=1}^{m} \alpha\left(2^{i}\right) & =\frac{2^{2^{m+2}-3}}{\pi^{m}} O\left(1-2^{-m}\right) \\
& =O\left(\frac{2^{2^{m+2}-3}}{\pi^{m}}\right) .
\end{aligned}
$$

Having disposed of the preliminary steps, write $\beta(i)=2^{m-i} \prod_{j=i}^{m} \alpha\left(2^{j}\right)$ so that our goal reduces to evaluating $\sum_{i=1}^{m} \beta(i)$. For $1 \leq i<m$, we first observe 
that

$$
\begin{aligned}
2 \beta(i+1) & =22^{m-i-1} \prod_{j=i+1}^{m} \alpha\left(2^{j}\right) \\
& =2^{m-i} \prod_{j=i+1}^{m} \alpha\left(2^{j}\right) \\
& \leq 2^{m-i} \prod_{j=i}^{m} \alpha\left(2^{j}\right) \quad(\text { since } \alpha(i) \geq 1) \\
& =\beta(i) .
\end{aligned}
$$

Therefore

$$
\begin{aligned}
\sum_{i=1}^{m} \beta(i) & \leq \sum_{i=1}^{m} \frac{\beta(1)}{2^{i-1}} \\
& =2 \beta(1) \sum_{i=1}^{m} 2^{-i} \\
& =2 \beta(1)\left(1-2^{-m}\right) \\
& \leq 2 \beta(1) .
\end{aligned}
$$

Then it follows that

$$
\begin{aligned}
\sum_{i=1}^{m} 2^{m-i} \prod_{j=i}^{m} \alpha\left(2^{j}\right) & \leq 2 \beta(1) \\
& =22^{m-1} \prod_{j=1}^{m} \alpha\left(2^{j}\right) \\
& =2^{m} O\left(\frac{2^{2^{m+2}-3}}{\pi^{m}}\right) \\
& =O\left(\frac{2^{2^{m+2}+m-3}}{\pi^{m}}\right)
\end{aligned}
$$

